\title{
A Courseware Development Methodology for Establishing Practice-Based Network Course
}

\author{
Jahwan Koo ${ }^{1}$ and Seongjin $\mathrm{Ahn}^{2}$ \\ 1 School of Information and Communications Engineering, Sungkyunkwan Univ. \\ Chunchun-dong 300, Jangan-gu, Suwon, Kyounggi-do, Korea \\ jhkoo@songgang.skku.ac.kr \\ 2 Department of Computer Education, Sungkyunkwan Univ. \\ Myeongnyun-dong 3-ga 53, Jongno-gu, Seoul, Korea \\ sjahn@comedu.skku.ac.kr*
}

\begin{abstract}
In this paper, we present a practice-based courseware development methodology for establishing a senior undergraduate network course for the computer-engineering department by reflecting on the rapidly changing information and communication technologies, enforcing practical education, and focusing on the existing and currently used curriculum models. Therefore, we have developed a special method, named it STM (Segmenting, Targeting, and Mapping), and applied it to the courseware development of a practice-based network course.
\end{abstract}

\section{Introduction}

Data communication and computer networks are major topics in the undergraduate computer-engineering curriculum. The goal of developing a courseware for network practice is to provide in-depth and meaningful networking content to teach senior undergraduate students. The first goal of this course is to teach the students how to comprehend and subsequently solve problems, specifically grasping the concepts of whole systems within the fields of network and communication systems. This is accomplished through the design and implementation, management, tuning and troubleshooting projects. The students can develop leadership skills through team projects. The second is to educate professionals in nationally competitive fields such as Internet, wired and wireless communication, home networking, and ubiquitous computing. The third is to diversify the contents of the course and the methods of instruction taking into consideration each student's ability, aptitude, and career path.

The courseware development for network practice lasted 12 months from March, 2003 to February, 2004. The objectives of this courseware are to educate students about internetworking technologies and simultaneously assist them in designing and building networks, and configure internetworking devices such as hubs, switches, routers, and wireless access points. The courseware development

\footnotetext{
^ Dr. S. Ahn is the Corresponding Author.
} 
team was composed of a professor in charge of the development, five M.S. and Ph. D's with relevant expertise and teaching experience, and a Cisco certified internetworking professional possessing the highest level of expertise.

The paper is organized as follows. Section 2 introduces a method, called STM (Segmenting, Targeting, and Mapping), which is used to design the courseware for network practice. Finally, conclusion is drawn in Section 3.

\section{$2 \quad$ STM(Segmenting, Targeting, and Mapping) Methodology}

At this point, there is an increasing need to prepare systematic procedures and methods for migrating principle-based into practice-based courses. Therefore, we have developed a special method referencing in [1] [2], named it STM, and applied it to the coureware development of a practice-based network course.

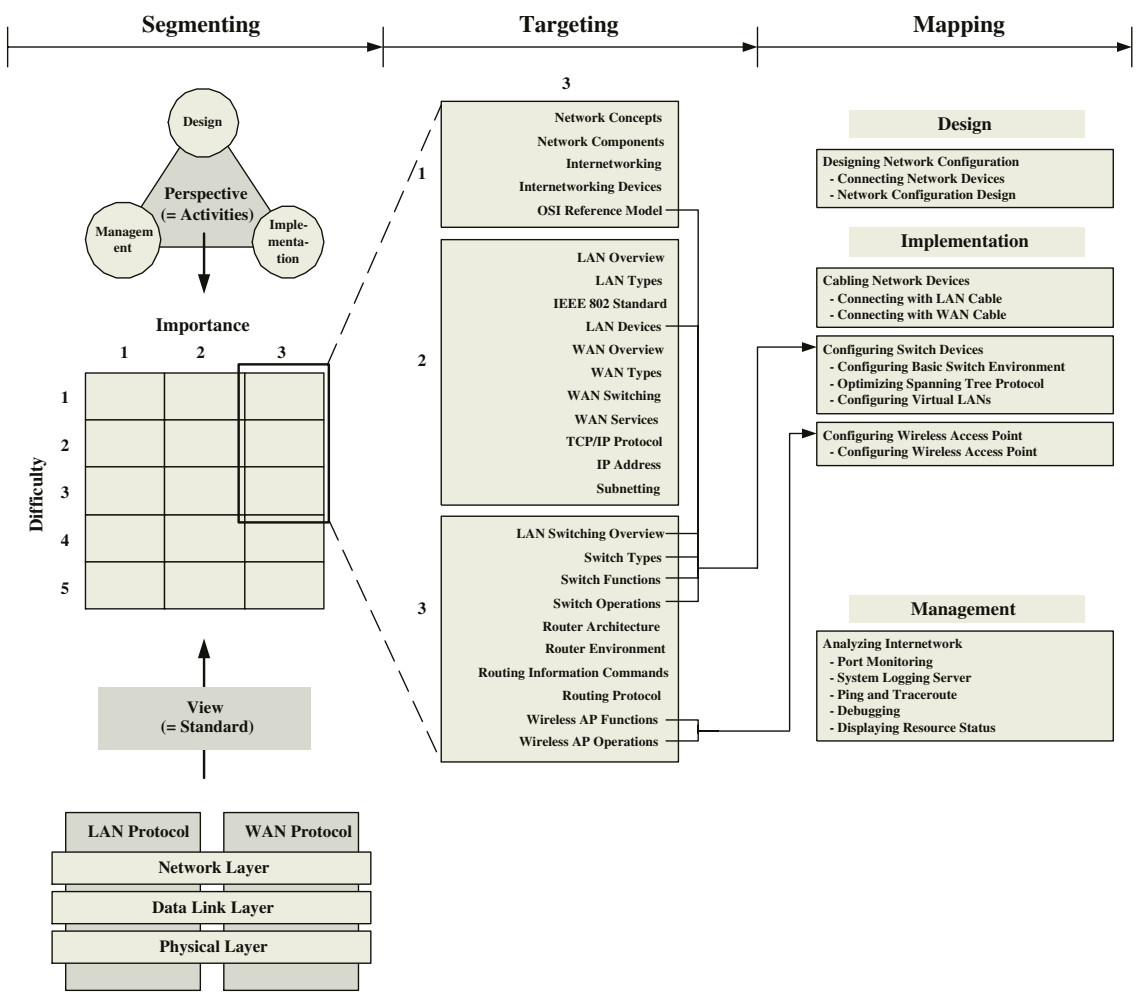

Fig. 1. STM diagram 


\subsection{Processes}

STM Methodology is divided greatly to 3 Processes: Segmenting, Targeting, and Mapping. Segmenting is to categorize the various topics according to the degree of the difficulty and the importance. For this process, we gathered categorized approximately 30 books and publications written on the internetworking technologies according to topics. We also referred to related books published by the Cisco Press, and e-learning materials by Cisco network academy program [3] [4] [5] [6]. Notably, the categorized topics could be altered according to various perspectives and different views. Figure 1 shows the case where a series of network activities and computer communication protocols are used as perspectives and views, respectively. The categorized topics were subsequently segmented by 5 levels of difficulty and 3 levels of importance, and were targeted in consideration of the educational purpose, students' ability, aptitude, and career choice. Next, the segmented and targeted topics were mapped into the contents for network practice by integrating single or multiple topics into inclusive contents.

\subsection{Output Definition}

Contents to be deduced in mapping process are inverted ultimately to the contents of the final output, for example, a student workbook and a supplement CD. The student workbook is the main textbook for both the instructors and students; it is divided into 5 Parts including exercises, 3 appendixes, and a glossary. The concepts covered in this textbook enable students to develop practical experience in skills related to configuring LANs, virtual LANs, static or dynamic routing protocols, and collecting and analyzing network traffic. In addition, this textbook extends students' practical experience with WANs, ISDN, PPP, Frame Relay design, configuration, and maintenance. In addition, this textbook is complemented by a supplement CD [7], which contains presentation slides for instructors, lab activity movies presented in a multimedia format, related references, and authors' e-mail addresses for students' questions. There are 34 lab activity movies in the supplement CD to deliver accurate information and enhance understanding about network activities performed in real world.

\subsection{Infrastructure Preparation: Network Lab Configuration}

To define step-by-step exercises for network practice, the network lab configuration diagram must be prepared. Such physical infrastructure is defined beforehand necessarily and should be examined because it can influence considerably content of teaching material or teaching material development costs. Figure 2 shows an example of the network lab configuration diagram. The network laboratory holds about 20 students, which is modelled from the hierarchical internetworking architecture composed of one main center, two regional centers, and four branch offices. Each center and branch office are connected by T1 or 64 Kbps leased lines. Personal computers on each branch office are connected to a 10/100 Mbps workgroup switch or a 802.11 b wireless access point. 


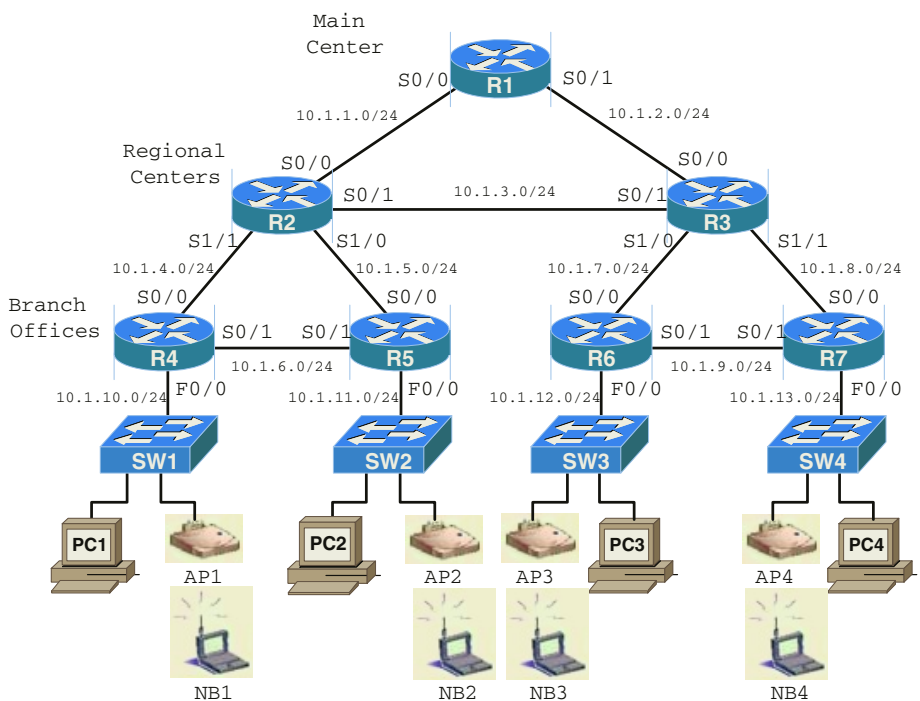

Fig. 2. Network lab configuration diagram

\section{Conclusion}

In this paper, we introduced the educational goals and development directions according to the change of curricula at SKKU's computer-engineering department. We presented the resources, people, time, and methods needed to develop a new courseware. We present the networking course as a proof-of-concept application of the STM methodology. In the future, we will gradually use this courseware in the network-centric courses.

\section{References}

1. J. T. Gorgone, G. B. Davis, J. S. Valacich, H. Topi, D. L. Feinstein, and H. E. Longenecker, "IS 2002 - Model Curriculum and Guidelines for Undergraduate Degree Programs in Information Systems," Association for Information Systems, 2002.

2. Computing Curricula 2001 Computer Science Final Report, December, IEEE-CS and ACM, 2001.

3. S. McQuerry, "Interconnecting cisco network devices," Cisco Systems, Inc, 2000.

4. Cisco networking academy program: first-year companion guide, Cisco Systems, Inc, 2001.

5. Cisco networking academy program: second-year companion guide, Cisco Systems, Inc, 2001.

6. Cisco networking academy program web site. [online] Available: http://cisco. netacad.net

7. Network practice course web site. [online] Available: http://songgang.skku.ac.kr/ jhkoo/index1.htm 